\title{
A Comparison of NBCA and RFA in Treating Varicose Veins in the Same Patient
}

\section{(1) Orhan Bozoğlan}

Gaziantep University Faculty of Medicine, Department of Cardiovascular Surgery, Gaziantep, Turkey

\begin{abstract}
Objectives: To compare N-butyl cyanoacrylate (NBCA) and radiofrequency ablation (RFA) in same patients with bilateral superficial venous insufficiency.

Materials and Methods: Sixty patients diagnosed with bilateral saphenous vein insufficiency between January 2017 and December 2018 were enrolled. Individuals with a saphenous vein diameter smaller than $5.5 \mathrm{~mm}$ at the saphenofemoral junction were excluded. The Clinical Severity, Etiology, Anatomy and Pathophysiology classification system was applied preoperatively. Venous clinical severity score values yielded by scoring preoperative clinical symptoms and findings were recorded. NBCA or RFA was applied to one leg, and the other procedure, either RFA or NBCA, was applied to the contralateral extremity.
\end{abstract}

Results: Minor complications observed with NBCA and RFA included induration at $20.7 \%$ and $31.0 \%$, ecchymosis at $31.0 \%$ and $51.7 \%$, and edema at $27.6 \%$ and $65.5 \%$, respectively. The recanalization rate in the NBCA group was $6.8 \%$, while no recanalization was observed in the legs undergoing RFA. Patient satisfaction rates were 51.7\% for NBCA and $31.0 \%$ for RFA, while $17.2 \%$ of patients were satisfied with both. Times to return to daily activity were 0.9 days in the NBCA group and 1.3 days in the RFA group. No statistically significant difference was observed between the groups in terms of procedural or postoperative pain. However, less pain was reported in the NBCA group in both periods $(\mathrm{p}<0.02)$.

Conclusion: NBCA may offer various advantages over RFA.

Keywords: Varicose veins, N-butyl cyanoacrylate, Radiofrequency ablation

Address for Correspondence: Orhan Bozoğlan, Gaziantep University Faculty of Medicine, Department of Cardiovascular Surgery, Gaziantep, Turkey

e-mail: orhanbozoglan1975@hotmail.com ORCID: orcid.org/0000-0002-5144-554X

Received: 18.02.2020 Accepted: 27.02.2020

Cite this article as: Bozoğlan O. A Comparison of NBCA and RFA in Treating Varicose Veins in the Same Patient.

EJCM 2020;8(1):45-50.

DOI: $10.32596 /$ jem.galenos.2020.02.09

${ }^{\circ}$ Copyright 2020 by Heart and Health Foundation of Turkey (TÜSAV) / E Journal of Cardiovascular Medicine published by Galenos Publishing House. 


\section{Introduction}

While surgery represented the main option in the treatment of varicose veins for many years, this has been replaced by endovenous methods in the last 20 years. The first procedure performed using radiofrequency energy was published in $2002^{(1)}$. Radiofrequency ablation (RFA) subsequently began being widely employed in varicose veins ${ }^{(2,3)}$. Thermal energy as high as 1200 $\mathrm{C}$ is used in RFA. However, tumescent anesthesia is essential in order to prevent potential complications associated with thermal energy. Various problems may also occur with tumescent anesthesia. Complications may develop in association with the use of high levels of local anesthetic agent. Ecchymosis and hematoma may also develop in the injection sites. The application of several injections may also be uncomfortable for the patient. The possibility of such complications in thermal ablation methods has previously been reported $^{(4,5)}$. In order to avoid the complications of tumescent anesthesia, there has recently been a direct trend toward non-thermal methods. Foam sclerotherapy was formerly used for that purpose, but has a low success rate $^{(6)}$. More effective methods have therefore been developed, such as mechanochemical ablation and glue embolization ${ }^{(7,8)}$. Glue ablation relies on chemical destruction of the insufficient vein using NBCA, a tissue adhesive. This technique has become highly popular in recent years and has begun being widely employed. Several studies involving NBCA have been published. Studies have also compared NBCA with other thermal ablation methods ${ }^{(8-11)}$. However, no previous studies have compared the two techniques in the same patient. The purpose of the present study was therefore to investigate procedural success, complications, and patient satisfaction in individuals undergoing NBCA and RFA to different legs. NBCA was applied to one lower extremity in the cases of bilateral saphenous vein insufficiency and RFA to the contralateral extremity in order to obviate patient-associated variables.

\section{Materials and Methods}

Sixty patients, including 26 men and 34 women aged between 29 and 64 years (mean $42.2 \pm 10.2$ years), diagnosed with symptomatic great saphenous vein insufficiency in the bilateral lower extremities and presenting to the cardiovascular surgery clinic between January 2017 and December 2018 represented the study group. The NBCA and RFA procedures in the present research were performed by two experienced surgeons in the same institution. NBCA was applied to one lower extremity and RFA to the other in all 60 cases. The study commenced following receipt of ethical committee approval. Patients with unilateral vena saphena magna (VSM) insufficiency, those undergoing the same technique in both extremities, and those refusing to consent to procedures on both extremities in separate sessions were excluded. We also excluded all individuals with saphenous vein diameters at the saphenofemoral junction (SFJ) smaller than $5 \mathrm{~mm}$. The Clinical Severity, Etiology, Anatomy and Pathophysiology (CEAP) classification system was applied prior to all procedures. Venous clinical severity score (VCSS) values were elicited by scoring preprocedural clinical symptoms and findings, and subsequently recorded. The decision to perform NBCA and RFA was based on the presence of insufficiency in both existing VSM identified at diagnostic colored Doppler ultrasonography (DUS). Evaluation revealed no advanced insufficiency or obstruction in the deep veins of either extremity in any individual. The procedures were randomized, with NBCA being performed first in one case and RFA first in the next. Patients were blinded to which procedure was scheduled for which extremity. NBCA and RFA were performed as previously reported by Yasim et al. ${ }^{(8)}$ and Eroglu and Yasim ${ }^{(9)}$. Analgesic medication (paracetamol) was made available for all patients after both NBCA and RFA. A pain scale was employed to measure pain occurring during and after the procedure. Patients were asked to indicate the level of pain perceived on a scale of 1 to 5, with 1 representing no pain, 2 mild pain, 3 moderate pain, 4 severe pain, and 5 very severe pain. 
All additional analgesia requirements were recorded. An elastic bandage was placed around the extremity receiving the procedure for two days. Patients were advised to wear compression socks for the next three months and to resume their daily activities as quickly as possible. Times to return to daily activities were also recorded. Clinical follow-up was performed on postprocedural day 2, and clinical and DUS-assisted follow-ups were carried out in the first week and at one, three, and six months. DUS was employed when recording saphenous vein occlusion, recanalization, perforating veins, and residual varicosities. Both major and minor complications were investigated.

The ethics committee approval was obtained from Kahramanmaraş Sütçü İmam University approved this study (REC number: 2018/14-05).

\section{Statistical Analysis}

Data were expressed as mean \pm standard deviation values or as median and range. Analysis of demographic and clinical data was performed using the paired samples t-test for parametric variables and the Wilcoxon signed ranks test for non-normally distributed data. The McNemar test was applied to analyze quantitative data. All analyses were performed on SPSS version 17.0 software (SPSS Inc., Chicago, USA), and $p$ values $<0.05$ were regarded as statistically significant.

\section{Results}

Primary VSM insufficiency was present in all patients. Both extremities of all patients were symptomatic. There was no difference between the patients in terms of CEAP and VCSS classifications. The mean duration of reflux in the SFJ was calculated as $3.6 \mathrm{~s}$ in the NBCA group and $3.8 \mathrm{~s}$ in the RFA group. NBCA and RFA procedures were applied to 120 saphenous veins of 60 patients. No significant differences were determined between the two groups in terms of saphenous vein diameters, lengths, or depths. However, the lengths of procedure differed significantly. NBCA was completed significantly at $21.2 \mathrm{~min}$, than RFA at 32.7 min $(p<0.05)$. A detailed comparison of demographic characteristics and clinical findings is shown in Table 1. Preoperative pain scores were 1.4 for NBCA and 1.7 for RFA, but the difference was not statistically significant. Postoperative pain scores were significantly lower in the NBCA group than in the RFA group $(\mathrm{p}<0.02)$. Postoperative analgesic requirements (paracetamol) were statistically significant. Time to return to activity was significantly shorter in the NBCA group than in the RFA group $(p<0.001)$. Time to return to work was not significantly different between the groups. A comparison of postoperative data is given in Table 2. Postoperative minor complications included induration, ecchymosis, and edema. Induration was observed in $20.7 \%$ of extremities receiving NBCA and $31.0 \%$ of those receiving RFA, the difference was statistically significant. Ecchymosis developed in $31.0 \%$ of extremities receiving NBCA and in $51.7 \%$ of those undergoing RFA. This difference was

Table 1. Demographic and clinical data

\begin{tabular}{|c|c|c|c|}
\hline & $\begin{array}{l}\text { NBCA } \\
(n=60)\end{array}$ & $\begin{array}{l}\text { RFA } \\
(n=60)\end{array}$ & $\mathbf{p}$ \\
\hline Age & $44 \pm 10.2$ & $44.2 \pm 10.2$ & - \\
\hline Gender, M/F & $28 / 32$ & $28 / 32$ & - \\
\hline vcss & $9.7 \pm 2.5$ & $9.9 \pm 2.5$ & $>0.05$ \\
\hline CEAP & $3.2 \pm 0.4$ & $3.2 \pm 0.4$ & $>0.05$ \\
\hline VSM diameter (SFJ) & $9.6 \pm 1.7$ & $10.3 \pm 2.8$ & $>0.05$ \\
\hline VSM diameter (knee) & $6.2 \pm 1.4$ & $6.4 \pm 2.3$ & $>0.05$ \\
\hline Mean SFJ reflux time (sec) & $3.6 \pm 1.4$ & $3.8 \pm 1.3$ & $>0.05$ \\
\hline Distance from skin (mm) & $14.3 \pm 7.3$ & $14.5 \pm 7.3$ & $>0.05$ \\
\hline Length of saphenous vein $(\mathrm{cm})$ & $26.4 \pm 3.4$ & $26.5 \pm 6.5$ & $>0.05$ \\
\hline Duration of procedure (min) & $21.2 \pm 4.7$ & $32.7 \pm 6.5$ & $<0.05$ \\
\hline \multicolumn{4}{|c|}{$\begin{array}{l}\text { NBCA: N-butyl cyanoacrylate, RFA: Radiofrequency ablation, VCSS. } \\
\text { Venous clinical severity score, CEAP: Clinical Severity, Etiology, Anatomy, } \\
\text { and Pathophysiology; VSM: Vena saphena magna, SFJ: Saphenofemora } \\
\text { junction, M: Male, F: Female } \\
\text { Bold } p \text { value are statistically significant }\end{array}$} \\
\hline
\end{tabular}

Table 2. Postoperative data

\begin{tabular}{|l|l|l|l|}
\hline & $\begin{array}{l}\text { NBCA } \\
(\mathbf{n = 6 0 )}\end{array}$ & $\begin{array}{l}\text { RFA } \\
(\mathbf{n = 6 0 )}\end{array}$ & $\mathbf{p}$ \\
\hline Pain score (intraoperative)/d & $1.2 \pm 0.6$ & $1.7 \pm 0.8$ & $<0.05$ \\
\hline Pain score (postoperative)/d & $1.2 \pm 0.4$ & $1.4 \pm 0.5$ & $<0.05$ \\
\hline Analgesic requirement, $\mathbf{m g} / \mathbf{d}$ & $650 \pm 300$ & $950 \pm 200$ & $<0.05$ \\
\hline Time to return to activity/d & $0.9 \pm 0.8$ & $1.3 \pm 1.1$ & $<0.05$ \\
\hline Time to return to work/d & $1.8 \pm 0.8$ & $2.1 \pm 1.2$ & $>0.05$ \\
\hline RFA: Radiofrequency ablation, NBCA: & N-butyl cyanoacrylate & \\
\hline
\end{tabular}


also significant. Edema developed in significantly fewer members of the NBCA group, at $27.6 \%$, than in the RFA group, at $65.5 \%(\mathrm{p}<0.008)$. All three minor complications resolved completely within two weeks. No major complications [such as deep venous thrombosis (DVT), pulmonary embolism, or skin burn] were observed in any case. Post-NBCA and RFA complications are shown in Table 3. In terms of satisfaction with the two procedures, $31 \%$ of patients reported favoring RFA while $51.7 \%$ preferred NBCA, and $17.2 \%$ expressed no preference. Recanalization occurred in four $(6.8 \%)$ saphenous veins undergoing NBCA during follow-up. Total occlusion was observed in all 60 (100\%) saphenous veins receiving RFA at six-month follow-up.

\section{Discussion}

Chronic venous insufficiency and resulting varicose veins in the lower extremities impact significantly on patients' quality of life and also cause socioeconomic burdens ${ }^{(12)}$. The last decade has witnessed major advances in the treatment of varicose veins, with endovenous ablation techniques replacing surgery to a significant extent. Thermal endovenous procedures including RFA EVLA have become the most commonly employed techniques. Cyanoacrylate is a generic name applied to a group of strong and fast-acting adhesives with industrial, medical, and domestic applications. NBCA, a type of adhesive, is approved by the Federal Drug Administration in the treatment of cerebral aneurysms and arteriovenous malformations ${ }^{(13-15)}$. Intra-body use is therefore safe for

Table 3. Complications After NBCA and RFA

\begin{tabular}{|l|l|l|l|}
\hline & $\begin{array}{l}\text { NBCA } \\
(\mathbf{n = 6 0 )}\end{array}$ & $\begin{array}{l}\text { RFA } \\
(\mathbf{n = 6 0 )}\end{array}$ & $\mathbf{p}$ \\
\hline Induration & $20.7 \%$ & $31.0 \%$ & $<\mathbf{0 . 0 5}$ \\
\hline Ecchymosis & $31.0 \%$ & $32.6 \%$ & $<\mathbf{0 . 0 5}$ \\
\hline Edema & $27.6 \%$ & $65.5 \%$ & $<\mathbf{0 . 0 5}$ \\
\hline Paresthesia & 0.0 & 0.0 & - \\
\hline Deep vein thrombosis & 0.0 & 0.0 & - \\
\hline Pulmonary embolism & 0.0 & 0.0 & - \\
\hline
\end{tabular}

RFA: Radiofrequency ablation, NBCA: N-butyl cyanoacrylate Bold $p$ value are statistically significant humans. The clinical use of cyanoacrylate-based materials for the treatment of varicose veins has also become increasingly popular. Early results were subsequently published by numerous researchers ${ }^{(8,16,17)}$, followed by mid-term results ${ }^{(18)}$. NBCA is a clear fluid that solidifies following a polymerization reaction once injected intravascularly and produces an inflammatory reaction in the venous wall ${ }^{(19-21)}$. The first study concerning the use of varicose vein treatment in humans was by Almeida et al. ${ }^{(22)}$ in 2013. Good results were obtained with NBCA in all these studies. Studies comparing NBCA with other endovenous methods also began being published. Morrison et al. ${ }^{(21)}$ first compared NBCA and RFA (the VeClose Study) and reported three-month results. Bozkurt and Y1lmaz ${ }^{(11)}$ subsequently compared NBCA with EVA and reported 12-month results. In 2017, Koramaz et al. ${ }^{(10)}$ published a study comparing NBCA and EVA, and in 2018 the VeClose study published its 24-month findings. Again in 2018, Yasim et al. ${ }^{(8)}$ and Eroglu and Yasim ${ }^{(9)}$. reported two-year results for 525 patients undergoing NBCA, EVLA and RFA. NBCA and other endovenous methods were found to be similarly effective in all these studies, although NBCA had the advantages of fewer side-effects and better patient comfort. However, different methods were employed in different patients in all these studies. Individual differences may therefore have affected the treatment outcomes. We employed the two different methods (NBCA and RFA) in the same patient in order to minimize these individual characteristics. Objective assessment may therefore be problematic when the two techniques are compared in different patients, due to individual differences in pain thresholds. The present study was therefore intended to compare the efficacy and side-effects of NBCA and RFA ablation by performing them in the same patient, but on different legs. Our scan of the literature revealed no previous research comparing the two techniques in this manner. Analysis revealed ablation rates of $100 \%$ for RFA and $93.2 \%$ for NBCA, the difference was statistically significant. Patient satisfaction was higher with NBCA, with better results also being observed for parameters including intraoperative and 
postoperative pain, postoperative analgesic requirements, resumption of daily activity, and return to work. Results for postoperative pain and time to resumption of daily activity were significantly better for NBCA $(p<0.035$ and $<0.001$, respectively). No procedure-associated major complication (DVT, pulmonary embolism, or skin burn) was observed in any of our cases, while minor complications were less common following NBCA. However, it is also important to remember that most minor complications (such as hematoma and ecchymosis) derive not from the procedural anesthesia administration that can reduce these side-effects to a minimum. The main differences between the two techniques will therefore consist of pain and occlusion rates, with lower pain scores being reported with NBCA.

\section{Conclusion}

The fact that NBCA and RFA were compared in the bilateral extremities of the same patients in this study minimized subject-dependent factors and permitted a more objective evaluation of patient satisfaction. In conclusion, while NBCA and RFA exhibit similar success rates, NBCA may offer a number of advantages over RFA.

\section{Ethics}

Ethics Committee Approval: The ethics committee approval was obtained from Kahramanmaraş Sütçü İmam University approved this study (REC number: 2018/14$05)$.

Informed Consent: Informed consent was obtained from all the patients.

\section{Peer-review: Externally peer-reviewed.}

Financial Disclosure: The author declared that this study received no financial support.

\section{References}

1. Weiss RA, Weiss MA. Controlled radiofrequency endovenous occlusion using a unique radiofrequency catheter under duplex guidance to eliminate saphenous varicose vein reflux: a 2-year follow-up. Dermatol Surg 2002;28:38-42.

2. Lurie F, Creton D, Eklof B, et al. Prospective randomized study of endovenous radiofrequency obliteration (closure procedure) versus ligation and stripping in a selected patient population (EVOLVeS Study). J Vasc Surg 2003;38:207-14.

3. Badham GE, Strong SM, Whiteley MS. An in vitro study to optimise treatment of varicose veins with radiofrequency-induced thermo therapy. Phlebology 2015;30:17-23.

4. Creton D, Pichot O, Sessa C, Proebstle TM; ClosureFast Europe Group. Radiofrequency-powered segmental thermal obliteration carried out with the ClosureFast procedure: results at 1 year. Ann Vasc Surg 2010;24:360-6.

5. Desmyttere J, Grard C, Wassmer B, Mordon S. Endovenous 980-nm laser treatment of saphenous veins in a series of 500 patients. J Vasc Surg 2007;46:1242-7.

6. Rasmussen LH, Lawaetz M, Bjoern L, Vennits B, Blemings A, Eklof B. Randomized clinical trial com-paring endovenous laser ablation, radiofrequency ablation, foam sclerotherapyand surgical stripping for great saphenous varicose veins. Br J Surg 2011;98:1079-87.

7. Elias S, Raines JK. Mechanochemical tumescentless endovenous ablation final results of the initial clinical trial. Phlebology 2012;27:67-72.

8. Yasim A, Eroglu E, Bozoglan O, Mese B, Acıpayam M, Kara H. A new nontumescent endovenous ablation method for varicose vein treatment: early results of n-butyl cyanoacrylate (variclose $\left.{ }^{\circledR}\right)$. Phlebology 2017;32:194-9.

9. Eroglu E, Yasim A. A Randomised Clinical Trial Comparing N-Butyl Cyanoacrylate, Radiofrequency Ablation and Endovenous Laser Ablation for the Treatment of Superficial Venous Incompetence: Two Year Follow up Results. Eur J Vasc Endovasc Surg 2018;56:553-60.

10. Koramaz İ, El Kılıç H, Gökalp F, et al. Ablation of the great saphenous vein with nontumescent n-butyl cyanoacrylate versus endovenous laser therapy. J Vasc Surg Venous Lymphat Disord 2017;5:210-5.

11. Bozkurt AK, Yılmaz MF. A prospective comparison of a new cyanoacrylate glue and laser ablation for the treatment of venous insufficiency. Phlebology 2016;31(1 Suppl):106-13

12. Evans CJ, Fowkes FG, Ruckley CV, Lee AJ. Prevalence of varicose veins and chronic venous insufficiency in men and women in the general population: Edinburgh Vein Study. J Epidemiol Community Health 1999;53:149-53

13. Linfante I, Wakhloo AK. Brain aneurysms and arteriovenous malformations: advancements and emerging treatments in endovascular embolization. Stroke 2007;38:1411-7.

14. Bruno CA, Meyers PM. Endovascular management of arteriovenous malformations of the brain. Interv Neurol 2012;1:109-23.

15. Ogilvy CS, Stieg PE, Awad I, et al; Special Writing Group of the Stroke Council, American Stroke Association. AHA scientific state-ment: recommendations for the management of intracra-nial arteriovenous malformations: a statement for healthcare professionals from a special writing group of the Stroke Council, American Stroke Association. Stroke 2001;32:1458-71.

16. Park I. Initial Outcomes of Cyanoacrylate Closure, VenaSeal System, for the Treatment of the Incompetent Great and Small Saphenous Veins. Vasc Endovascular Surg 2017;51:545-9.

17. Çalık ES, Arslan Ü, Ayaz F, et al. N-butyl cyanoacrylate in the treatmen of venous insufficiency--the effect of embolisation with ablative polymerisation. Vasa 2016;45:241-6. 
18. Levrier O, Mekkaoui C, Rolland PH, et al. Efficacy and low vascular toxicity of embolization with radical versus anionic polymerization of N-butyl-2-cyanoacrylate (NBCA): an experimental study in the swine. J Neuroradiol 2003;30:95-102.

19. Eroglu E, Yasim A, Ari M, et al. Mid-term results in the treatment of varicose veins with N-butyl cyanoacrylate. Phlebology 2017;32:665-9.

20. Levrier O, Mekkaoui C, Rolland PH, et al. Efficacy and low vascular toxicity of embolization with radical versus anionic polymerization of
N-butyl-2-cyanoacrylate (NBCA): an experimental study in the swine. J Neuroradiol 2003;30:95-102.

21. Morrison N, Gibson K, McEnroe S, et al. Randomized trial comparing cyanoacrylate embolization and radiofre-quency ablation for incompetent great saphenous veins (VeClose). J Vasc Surg 2015;61:985-94.

22. Almeida JI, Javier JJ, Mackay EG, Bautista C, Cher DJ, Proebstle TM. Two-year follow-up of first human use of cyanoacrylate adhesive for treatment of saphenous vein incompetence. Phlebology 2015;30:397-404. 